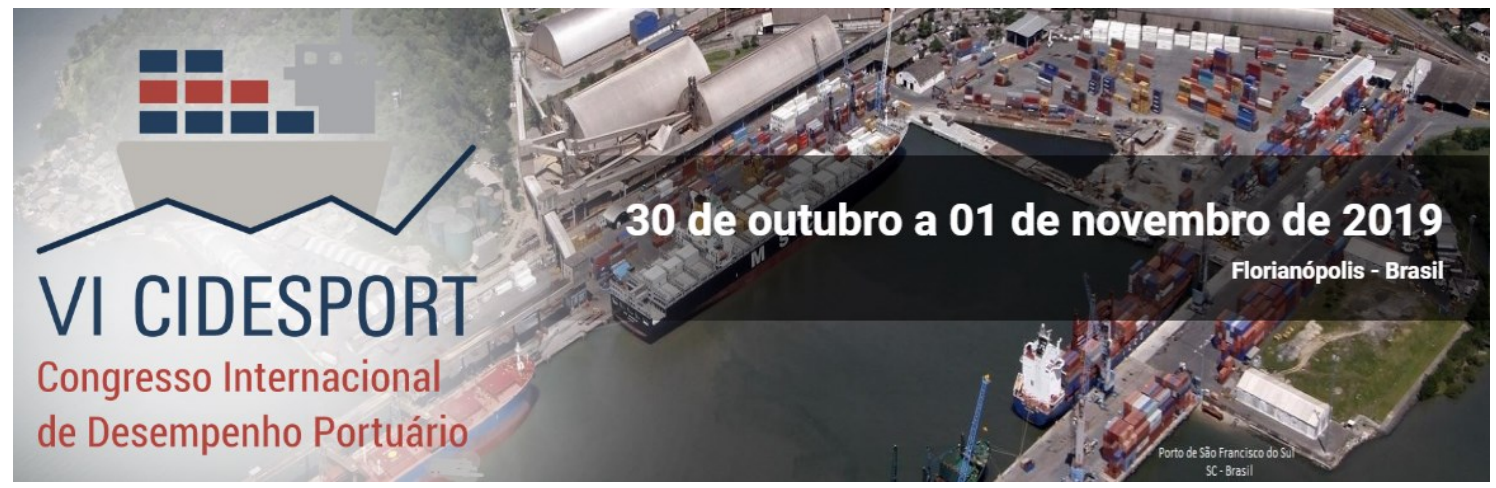

\title{
SEQUENCIAMENTO DE OPERAÇÕES PORTUÁRIAS COM CONFLITO UTILIZANDO A METAHEURÍSTICA GRASP
}

\section{Gustavo Campos Menezes Centro Federal de Educação Tecnológica de Minas Gerais \\ Raphael de Assis Silva Centro Federal de Educação Tecnológica de Minas Gerais}

Resumo: Em diversas aplicações há a necessidade de agendar atividades a serem executadas e deseja-se obter um agendamento que minimize o tempo gasto para concluí-las. Quando essas atividades possuem conflitos entre si, isto é, algumas atividades não podem ser executadas ao mesmo tempo que outras, temos um problema complexo. Uma especificação desse problema ocorre em sistemas de transportes como terminais portuários. Os terminais portuários apresentam um sistema de rotas de cargas muito complexo e que possibilita uma grande quantidade de combinações possíveis. Com isso, surge a necessidade de otimizar essas rotas em busca de um caminho mínimo, reduzindo assim o tempo para carregar e descarregar os navios. O objetivo desse estudo é buscar soluções Heurísticas baseadas na metaheurística GRASP para reduzir o makespan em casos gerais onde operações com conflitos devem ser agendadas.

Palavras chave: Logística portuária. Sequenciamento da Produção. Heurísticas. Otimização

\section{INTRODUÇÃO}

O grande avanço no setor de transportes deu início a uma verdadeira revolução no processo de globalização. A demanda por capacidade e agilidade em entregas tornou os sistemas de transporte, incluindo o naval, extremamente complexo, demandando diariamente milhares de toneladas de produtos a serem transportados. Os terminais portuários são responsáveis por carregar e descarregar navios de carga e fornecer suporte de armazenamento para intermediar a troca da carga com outros meios de transporte como caminhões e trens. Devido a enorme quantidade de produtos carregados pelos navios a carga e descarga se torna extremamente demorada e torna bastante trabalhoso alocar os pátios de armazenamento para os produtos. Por esses motivos é de grande relevância que seja desenvolvido meios de otimizar as rotas dos produtos e o processo de alocação dos pátios, reduzindo o tempo para descarregar os navios. Em terminais portuários que transportam produtos a granel, os pátios de armazenamento dos produtos precisam ser lavados para receber um produto diferente, e nesse caso um

${ }^{*}$ A revisão gramatical, ortográfica, ABNT ou APA foi realizada pelos autores. 
agendamento que gere menos trocas de produtos no mesmo pátio economiza tempo e dinheiro com a limpeza desses locais. Assim, esses fatores motivam a busca de um algoritmo que gere um bom agendamento de rotas com conflitos.

Tratamos nesse estudo um problema geral de sequenciamento de tarefas que apresentem conflitos entre si. A partir disso, busca-se desenvolver um algoritmo capaz de otimizar o agendamento das atividades com conflitos, reduzindo o tempo necessário para a conclusão da tarefa. Exploraremos o problema prático do agendamento de rotas de transporte de carga em terminais portuários como particularização desse problema.

\section{DETALHAMENTO DO PROBLEMA DO PORTO}

A abstração do problema é inspirada no processo de carga e descarga de navios em terminais portuários. O desafio na escolha de rotas e alocação dos pátios nos portos agrupam problemas de grande complexidade devido à enorme possibilidade de agendamentos e à descontinuidade das soluções. Os portos possuem uma grande quantidade de rotas para transportar os produtos e várias dessas rotas compartilham os mesmos equipamentos, impossibilitando a execução de ambas rotas simultaneamente.

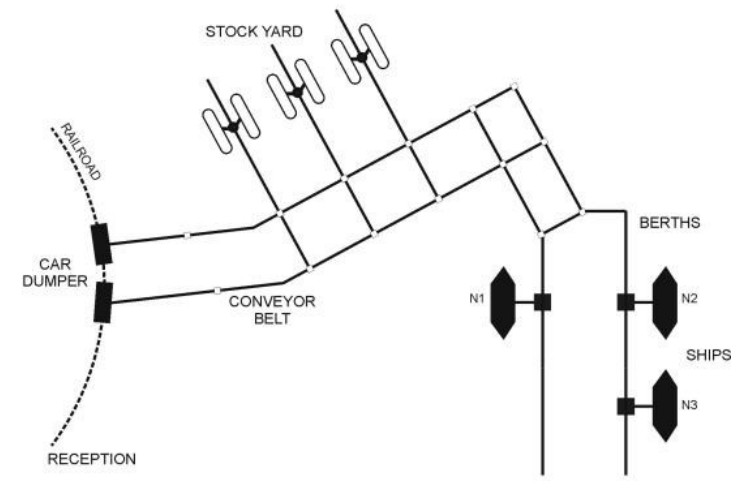

Figura 1. Representação de um terminal portuário

Na figura 1 é representado o esquema de um terminal portuário. Os navios atracam no píer e aguardam para serem carregados ou descarregados. As rotas para transporte dos produtos são provenientes do sistema ferroviário ou pátio de armazenamento. Em diversos momentos ocorrem conflitos entre as rotas, isto é, ambas necessitam de um mesmo equipamento para completar sua rota, como é representado na figura 2. Nesses casos um dos produtos deve aguardar o outro ser transportado para em seguida poder continuar a sua rota. 
VI CIDESPORT/2019

Congresso Internacional

de Desempenho Portuário
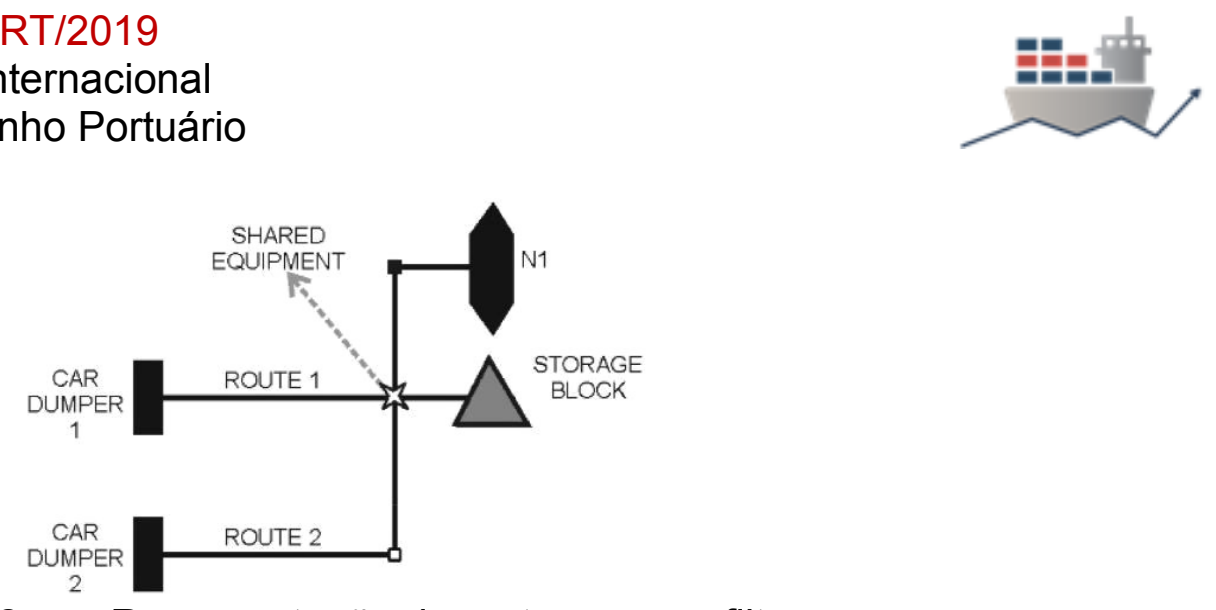

Figura 2. Representação das rotas em conflito

Além da complexidade em evitar os conflitos é desejável gerar um agendamento em que ocorra menos trocas de produtos nos pátios de armazenamento. Isto ocorre pois em portos que trabalham com produtos a granel, como soja, milho e demais grãos, é preciso lavar os pátios para armazenar outro produto no local. O mesmo ocorre com produtos do mesmo tipo e de purezas diferentes. Nas próximas seções será abordado a modelagem do problema e será apresentado o algoritmo desenvolvido.

\section{REVISÃO DA LITERATURA}

Nas últimas décadas, diversos autores, entre eles (Christopher, 1998) e (Shapiro, 2006), apontam a informação como o principal ativo de uma empresa. É crescente a integração de empresas, países e pessoas, através dos mecanismos de comunicação. $\mathrm{O}$ crescimento do comércio internacional e a busca pela qualidade, produtividade e redução de estoques, pressionam cada vez mais os profissionais responsáveis pela tomada de decisão das empresas.

A Pesquisa Operacional e o uso da Tecnologia da Informação tornaram-se ferramentas importantes para estes profissionais conseguirem obter os resultados desejados em suas organizações. Para procurar atender os objetivos acima, as empresas dividem sua estrutura de decisão em três níveis: nível estratégico (planejamento de longo prazo), nível tático (planejamento de médio prazo) e nível operacional (programação de curto prazo).

Os grandes desafios envolvidos na integração das operações de produção e logística motivam pesquisas voltadas para aumento da produtividade, redução de custos e geração de valor nos empreendimentos. Nesta linha de pesquisa, diversos trabalhos como os de (Bichou,2004), (Singh, 2012) e (Pimentel et al ,2013), destacam a necessidade de soluções integradas que levem em consideração os problemas associados aos três níveis: estratégico, tático e operacional. Neste artigo são investigadas soluções integradas para um problema que envolve os níveis tático e operacional.

A pesquisa pela integração entre os níveis de decisão não é uma ideia nova. Vários esforços já foram feitos buscando realizar este desafio como em (Meyr,2000), (Gaglioppa et al, 2008), (Ferreira et al, 2010), (Mateus et al, 2010) e, mais recentemente, com (Kis e Kovács,2012). Entretanto, realizar esse procedimento não é uma tarefa trivial. Os problemas obtidos a partir desta integração são conhecidamente complexos. A coerência entre os dados nos dois níveis não é facilmente garantida e, em boa parte, não se 
VI CIDESPORT/2019

Congresso Internacional

de Desempenho Portuário

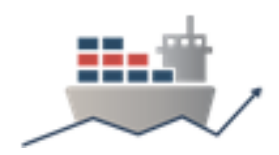

consegue representar com grande sucesso os problemas reais das empresas.

\section{REPRESENTAÇÃO MATEMÁTICA DO PROBLEMA}

O modelo matemático proposto para o problema é utilizar um grafo de conflito para representar as incompatibilidades das rotas, onde os vértices representam as rotas dos produtos e as arestas representam que existe um conflito entre elas.

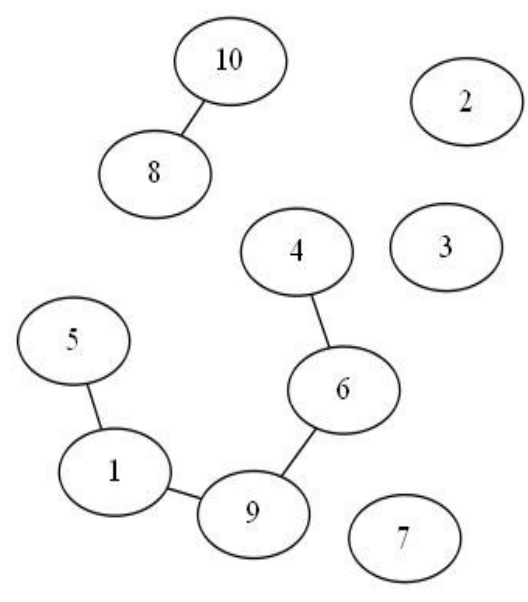

Figura 3. Representação de um grafo de conflito

Na figura 3 há o exemplo de um grafo de conflitos de 10 vértices. Os vértices são enumerados de 1 a 10 e há arestas ligando alguns desses vértices. $O$ grafo da figura representa que as rotas 2, 3 e 7 não possuem conflito com nenhuma outra, ou seja, podem ser realizadas a qualquer momento pois as máquinas que iram operá-las não serão utilizadas por nenhuma outra rota. Por outro lado, as rotas 10 e 8 não podem ser utilizadas ao mesmo tempo devido ao fato de compartilharem o mesmo equipamento, o que exige que uma rota só seja realizada após o término da outra. O mesmo acontece com as rotas 1 e 5 ; 1 e 9 ; 6 e 9 ; e 4 e 6 que também possuem conflitos entre si. Um exemplo de solução para esse problema representado na figura 3 seria agendar as rotas $2,3,7,5,9,4$ e 10 para iniciarem no tempo zero. Quando a rota 10 terminar iniciar imediatamente a 8. Após as rotas 5 e 9 terminarem iniciar a rota 1 e quando as rotas $4 \mathrm{e}$ 9 terminarem iniciar a rota 6 . Note que se optássemos por realizar as rotas 1 e 6 primeiros deixaríamos as rotas 4, 5 e 9 em espera, o que, em primeira análise, sugere um tempo maior para o transporte.

Os conflitos serão medidos em termos de densidade e essa medida é feita calculando a razão entre o número de arestas no grafo de instancia pelo número de arestas de um grafo completo com o mesmo número de vértices.

Uma vez escolhido o modelo do problema analisamos o comportamento das soluções para diferentes quantidades de rotas e conflitos. Variando-se o número de rotas percebemos um aumento na complexidade do problema, pois assim mais possibilidades de agendamento serão possíveis. Cada agendamento terá rotas operando em paralelo e 
rotas em espera que dependerão da finalização de outras para serem executadas. Desse modo, em algum agendamento pode ocorrer de uma rota com um número elevado de conflitos ser selecionada para iniciar no tempo zero, deixando muitas outras rotas em espera. Por outro lado, um segundo agendamento poderia selecionar as rotas com menos conflitos para iniciarem, evitando o travamento de muitas rotas.

Analisando o problema quando se varia a densidade de conflitos notamos uma tendência no aumento da complexidade do problema. Quando a densidade de conflitos é muito pequena ou muito grande o agendamento é mais simples. No caso de não haver conflitos todas as rotas podem ser agendadas para começar no tempo zero e o makespan seria o tempo demandado pela rota mais demorada. Por outro lado, no caso em que todas as rotas tiverem conflitos entre si todas as possibilidades de agendamento possuirão o mesmo makespan, a soma dos tempos de execução de todas as rotas, pois só poderá haver uma única rota em funcionamento a cada momento, tornando trivial a solução. Esse comportamento de mantém para densidades não muito distantes dos extremos, pois com poucos conflitos muitas rotas irão estar operando em paralelo e com muitos conflitos poucas rotas estarão em funcionamento ao mesmo tempo, o que limita as alterações que podem ser feitas no agendamento. Por esse motivo o maior desafio se encontra em realizar agendamentos em que a densidade de conflitos entre as rotas é intermediária. A seguir é ilustrado dois agendamentos distintos para o mesmo problema nas figuras 4 e 5 . Nos diagramas o eixo horizontal representa o tempo e o eixo vertical representa a rota. As linhas horizontais marcam o tempo de inicio e fim de cada rota respectivamente.

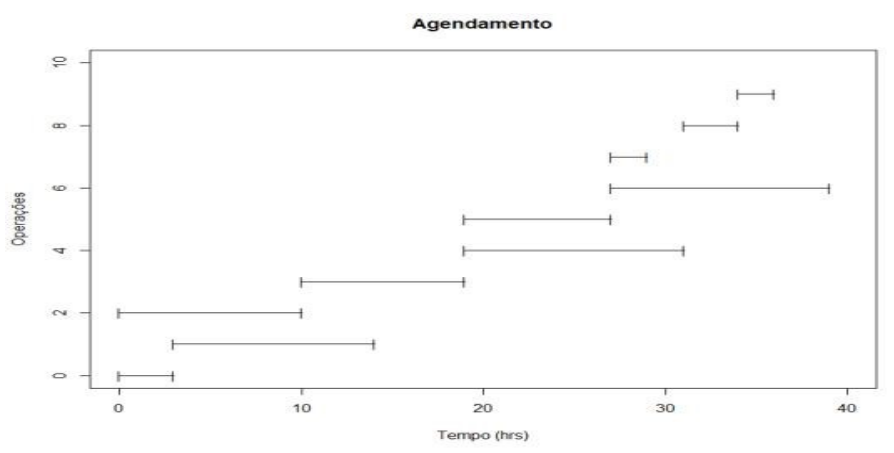

Figura 4.Agendamento Inicial

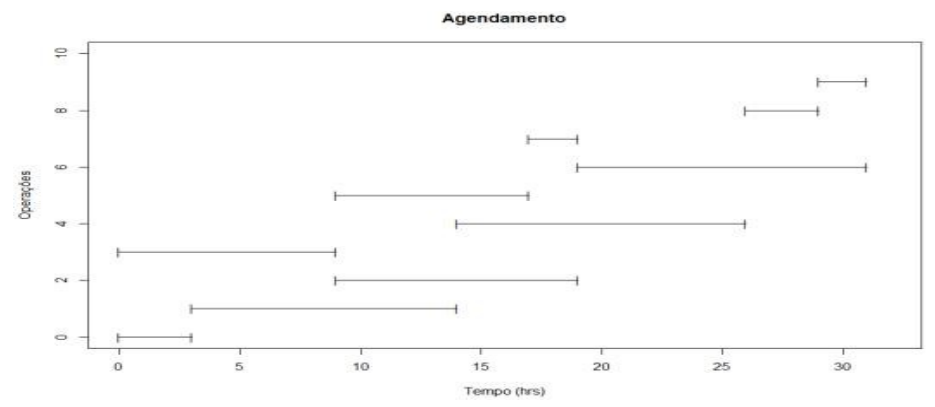

Figura 5. Agendamento Otimizado 
VI CIDESPORT/2019

Congresso Internacional

de Desempenho Portuário

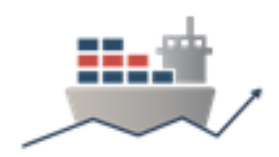

É possível observar um makespan significativamente menor quando executamos as rotas na ordem especificada pela figura 5 .

\section{ALGORITMO PROPOSTO}

Para o problema abordado é proposto a utilização da meta-heurística GRASP (Greedy Randomized Adaptive Search Procedure). O GRASP consiste em gerar uma construção gulosa para o problema e em seguida gerar perturbações na solução em busca de melhorar o resultado encontrado. Essa perturbação na solução é repetida até um limite de iterações seja atingido. O pseudocódigo do GRASP está representado abaixo.

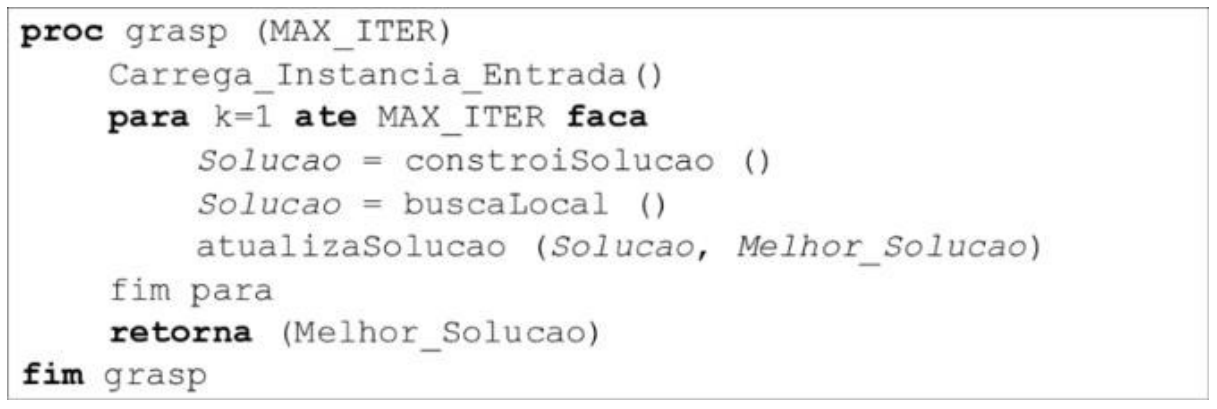

Figura 7. Exemplo Grasp

A ideia da heurística é esperar que as perturbações realizadas pela busca local diminuam o makespan e a partir do momento que a solução gerada deixar de melhorar, espera-se ter encontrado um mínimo local. Na figura 8 o eixo das abscissas representa o agendamento e o valor da abscissa representa o índice do agendamento. $O$ eixo das ordenadas representa o makespan encontrado para a solução com aquele agendamento respectivo. Essa relação não se trata de uma função, pois não há uma relação bem definida entre a pertubação gerada pela busca local e o makespan encontrado.

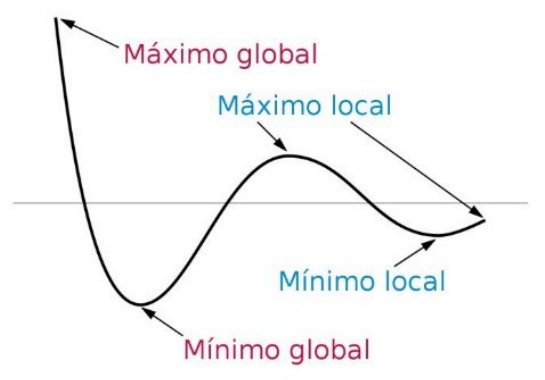

Figura 8 Perturbações na solução

A estratégia da heurística está em tentar desenvolver uma busca local que perturbe a solução de forma a cada iteração encontrar um makespan menor. 
O algoritmo desenvolvido inicia preenchendo uma estrutura de dados que contém as rotas e seus tempos de início e fim. De início, para a construção gulosa, busca-se entre as rotas aquelas que não possuem conflitos e as agenda para o começo. Em seguida, caso a rota possua algum conflito, busca-se entre as rotas conflituosas a que termina por último e agenda a nova rota após seu término. Para a primeira fase de busca local, realiza-se a alteração das rotais que iniciam no tempo zero, gerando assim uma perturbação na solução encontrada pela função de construção. A função GRASP, como dito anteriormente, inicia a construção gulosa da solução e em seguida perturba a solução encontrada em busca de um ótimo local, isto é, busca encontrar uma solução melhor que a anterior. Para aumentar as chances de sucesso implementamos três funções de busca local visando encontrar máximos locais de várias maneiras e uma função busca geral para encontrar uma solução melhor fora da vizinhança.

Como o resultado obtido pela construção não possui nenhuma garantia de otimalidade é preciso tratar essa solução para garantir um resultado melhor. Como foi mostrado no exemplo da figura 3 uma das formas de melhorar a solução é mudando a ordem das atividades selecionadas e assim liberamos outras atividades em um momento anterior, gerando um novo makespan. As funções de busca local apenas alteram a ordem de duas atividades e agendam novamente todas as outras atividades de acordo com seus conflitos. O pseudocódigo dos algoritmos é apresentado a seguir.

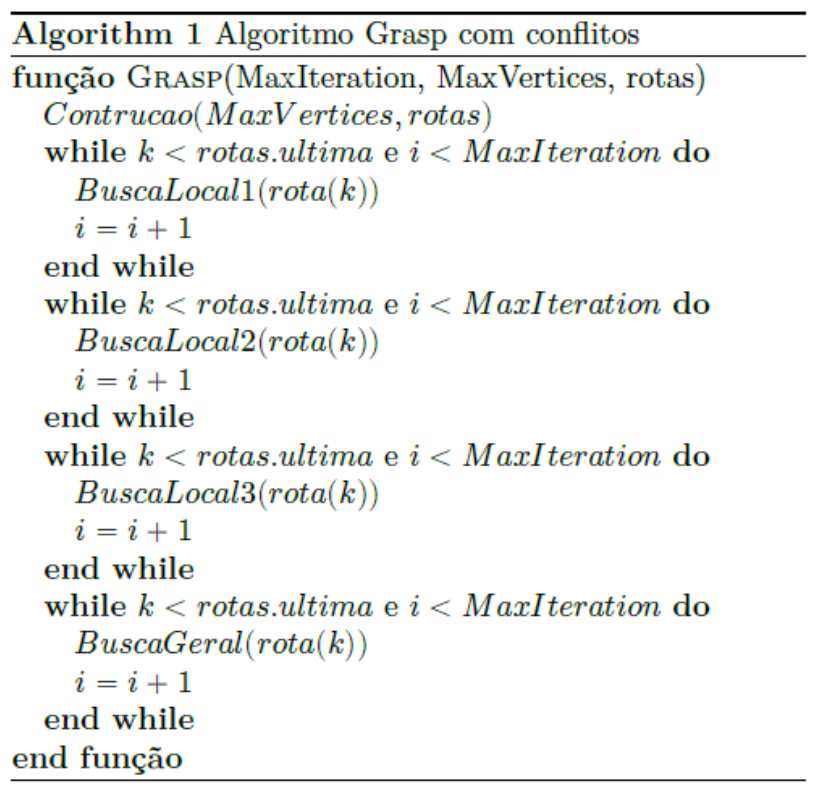


VI CIDESPORT/2019

Congresso Internacional

de Desempenho Portuário

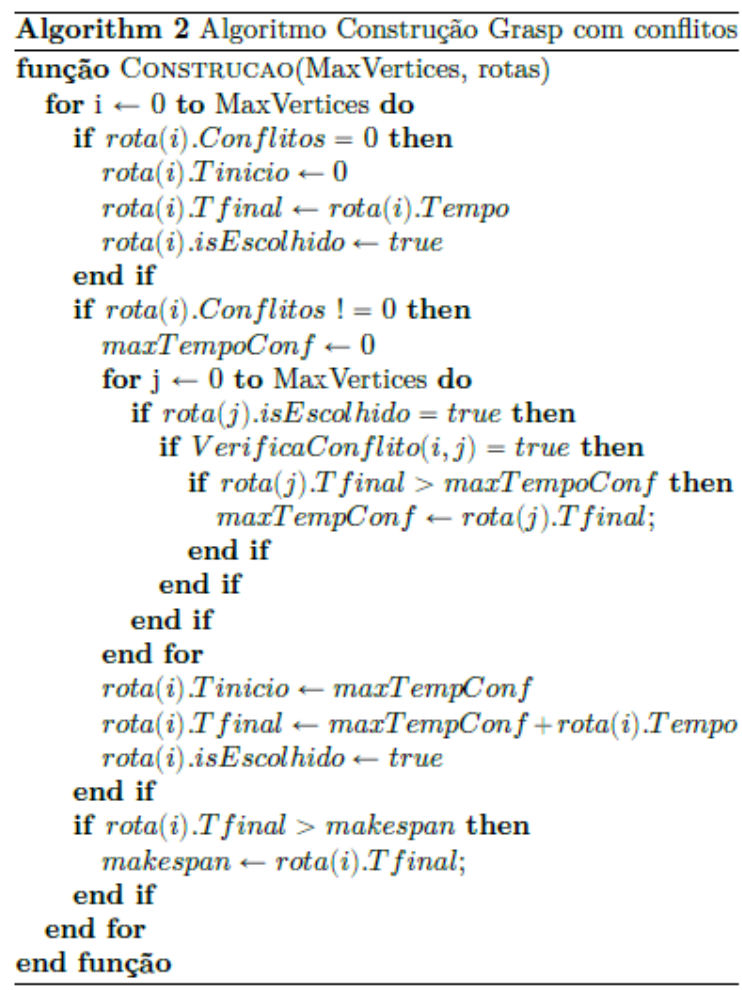

\section{EXPERIMENTOS COMPUTACIONAIS}

A partir do algoritmo desenvolvido foram realizados testes com várias instâncias aleatórias. As instancias foram criadas com quantidades crescentes de vértices e conflitos, tornando assim mais difícil de encontrar a solução ótima. A imagem da figura 9 mostra um exemplo de instancia utilizada nos testes.

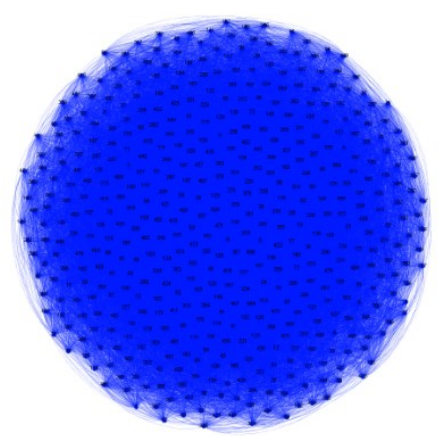

Figura 9. Exemplo de instância. 500 Vértices e $20 \%$ de conflitos

Os testes foram realizados em um notebook com processador intel i7 7 geração e $8 \mathrm{~Gb}$ de RAM. O tempo de execução do programa foi medido utilizando a função gettimeofday da biblioteca "sys/time.h". O tempo de solução para cada instancia está listado na tabela abaixo. 
VI CIDESPORT/2019

Congresso Internacional

de Desempenho Portuário

Tabola I

Tempo de execução

\begin{tabular}{|c|c|c|}
\hline Instancia & $\%$ Conflitos & Tempo Grasp $(\mu \mathrm{s})$ \\
\hline 10 & 10 & 1 \\
\hline 10 & 25 & 1 \\
\hline 10 & 50 & i \\
\hline 20 & 10 & 1 \\
\hline 20 & 25 & 1 \\
\hline 20 & 50 & 1 \\
\hline 50 & 10 & 994 \\
\hline 50 & 25 & 1993 \\
\hline 50 & 50 & 1995 \\
\hline 100 & 10 & 10996 \\
\hline 100 & 25 & 13995 \\
\hline 100 & 50 & 17976 \\
\hline 500 & 10 & 1149956 \\
\hline 500 & 25 & 1677513 \\
\hline 500 & 50 & 2092408 \\
\hline 1000 & 10 & 9553490 \\
\hline 1000 & 25 & 14134210 \\
\hline 1000 & 50 & 17598947 \\
\hline 5000 & 10 & inxiáxel \\
\hline 5000 & 25 & inxiáxel \\
\hline 5000 & 50 & inxiável \\
\hline
\end{tabular}

\section{CONCLUSÃO}

Neste artigo foi abordado um importante problema de tomada de decisão relacionado ao planejamento e sequenciamento de produtos em terminais portuários graneleiros. Foi destacado o problema de sequenciamento com restrições de incompatibilidade e desenvolvido uma heurística capaz de resolvê-lo. Sobre os experimentos, conclui-se que a heurística foi efetiva. Entretanto, mais estudos sobre o método e a avaliação de abordagens exatas para o problema precisam ser investigados.

\section{Agradecimentos}

Esta pesquisa foi patrocinada pelas seguintes instituições: Fundação de Amparo a Pesquisa do Estado de Minas Gerais (FAPEMIG) e Conselho Nacional de Desenvolvimento Científico e Tecnológico (CNPq).

\section{REFERÊNCIAS}

Christopher, M. (1998). Logistics and supply chain management:strategies for reducing cost and improving service. Prentice Hall.

Shapiro., J. F. (2006). Modeling the supply chain. 2nd ed.Duxbury Press.

Bichou, K. \& Gray, R. (2004). A logistics and supply chain management approach to 
VI CIDESPORT/2019

Congresso Internacional

de Desempenho Portuário

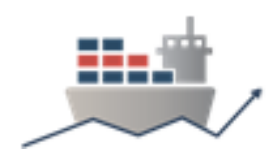

port performance measurement. Maritime Policy \& Management, 31(1):47_67.

Singh, G.; Sier, D.; Ernst, A. T.; Gavriliouk, O.; Oyston, R.; Giles, T. \& Welgama, P. (2012). A mixed integer programming model for long term capacity expansion planning: A case study from the hunter valley coal chain. European Journal of Operational Research, 220(1):210_ 224.

Pimentel, B. S.; Mateus, G. R. \& Almeida, F. A. (2013). Stochastic capacity planning and dynamic network design. International Journal of Production Economics,145(1):139 _ 149.

Meyr, H. (2000). Simultaneous lotsizing and scheduling by combining local search with dual reoptimization. European Journal of Operational Research, 20(2):311_326.

Gaglioppa, F.; Miller, L. A. \& Benjaafar, S. (2008). Multitask and multistage production planning and scheduling for process industries. Operations Research, 56(4):1010_1025.

Ferreira, D.; Morabito, R. \& Rangel, S. (2010). Relax and fix heuristics to solve one-stage one-machine lot-scheduling models for small-scale soft drink plants. Computers \& Operations Research, 37(4):684--691.

Mateus, G. R.; Ravetti, M. G.; Souza, M. C. \& Valeriano, T. M. (2010). Capacitated lot sizing and sequence dependent setup scheduling: an iterative approach for integration. Journal of Scheduling, 13(3):245_259.

Kis, T. \& Kovács, A. (2012). A cutting plane approach for integrated planning and scheduling. Computers and Operations Research, 9(2):320_327.

UNCTAD (2012). Review of maritime transport. Relatório técnico, United Nations Conference on Trade and Development, http://www.unctad.org.

Vacca, I.; Salani, M. \& Bierlaire, M. (2013). An exact algorithm for the integrated planning of berth allocation and quay crane assignment. Transportation Science,47(2):148_161.

Barros, V. H.; Costa, T. S.; Oliveira, A. C. M. \& Lorena, L. A. N. (2011). Model and heuristic for berth allocation in tidal bulk ports with stock level constraints. Computers \& Industrial Engineering, 60(4):606_613.

Angelelli, E., Kalinowski, T., Kapoor, R., Savelsbergh, M.W.: A reclaimer scheduling problem arising in coal stockyard management. J. of Scheduling 19(5), 563-582 (2016).

Menezes, G.C., Mateus, G.R., Ravetti, M.G.: A branch and price algorithm to solve the integrated production planning and scheduling in bulk ports. European Journal of Operational Research 258(3), 926 - 937 (2017).

Menezes, G.C., Mateus, G.R., Ravetti, M.G.: A hierarchical approach to solve a production planning and scheduling problem in bulk cargo terminal. Computers \& Industrial Engineering 97, 1-14 (2016). 